\title{
PROOF THAT WEBER'S NORMAL UNITS ARE NOT PERFECT POWERS
}

\author{
HARVEY COHN ${ }^{1}$
}

1. Introduction. In this paper the so-called Weber normal unit $\tau=\tan \pi / m$, in the field $R(2 \cos 2 \pi / m)$ where $m=2^{k} \geqq 8$, is shown to be unequal to an odd power of another integer in the field (even powers being excluded by trivial sign considerations).

The value of this result lies in the fact that the occurrence of an exact power with (odd) exponent $q$ would imply that the class number of the field had the divisor $q^{m / 8}$ according to the classic work of Weber [2].

In another paper, a large scale computation [1] was conducted to discover possible divisors of the class number of the fields of type $R(2 \cos 2 \pi / m)$. This computation, for simplicity, ignored contributions to the class number based on $\tau$ being an exact $q$ power. Reconsidering that work in conjunction with the present paper, we can conclude that none of these fields have a class number divisible by a prime less than 257 . The present work, however, is completely independent of the computation to which we just referred.

2. The trace. We write our field as $R(\zeta+1 / \zeta)$ where $\zeta=\exp 2 \pi i / m$. It is of degree $m / 4$ and is abelian having its (equal) conjugate fields generated by the cyclic transformations $\zeta^{s} \rightarrow \zeta$ where $s=5^{t}$ and $0 \leqq t$ $<m / 4$. Since we are not concerned with Galois structures, we can write the transformations as

$$
\zeta^{4 u+1} \rightarrow \zeta, \quad 0 \leqq u<m / 4 .
$$

A basis of the field is $1,(\zeta+1 / \zeta),\left(\zeta^{2}+1 / \zeta^{2}\right), \cdots,\left(\zeta^{m / 4-1}+1 / \zeta^{m / 4-1}\right)$, for instance. Thus it is evident that, since the defining equation of the specific number $\zeta$ is

$$
\zeta^{m / 2}+1=0,
$$

then the trace of any power of $\zeta$ is 0 except when (trivially) the power of $\zeta$ equals \pm 1 . Hence any integer in $R(\zeta+1 / \zeta)$ has a trace which is an integral multiple of the degree of the field, $m / 4$.

We next show that $\tau$ has trace $-m / 4$. Here, from equation (1), $\tau$ is one conjugate of the set

Presented to the Society, November 19, 1960 under the title Proof that Weber's normal unit is no perfect power; received by the editors November 14, 1960.

${ }^{1}$ Research supported by National Science Foundation Grant G-7412. 


$$
\tau_{u}=\tan (4 u+1) \pi / m, \quad 0 \leqq u<m / 4,
$$

and the trace $S(\tau)$ is the sum of all these conjugates. More conveniently, we note

$$
\begin{aligned}
\tau_{u} & =\left(\zeta^{4 u+1}-1\right) / i\left(\zeta^{4 u+1}+1\right) \\
& =(\zeta \omega-1) / i(\zeta \omega+1)
\end{aligned}
$$

where $\omega=\zeta^{4 u}=\exp 8 \pi i u / m$ a general root of the equation

$$
\omega^{m / 4}=1 \text {. }
$$

Thus

$$
S(\tau)=\sum_{\omega}(\zeta \omega-1) / i(\zeta \omega+1) .
$$

To evaluate the sum we consider the following integral

$$
I=\frac{1}{2 \pi i} \int \frac{\zeta z-1}{i(\zeta z+1)} \frac{d z}{z\left(z^{m / 4}-1\right)}
$$

where the path is a circle of radius larger than 1 . By deformation to $\infty, I=0$, while the integrand displays the residue $-i$ at $z=0$, the residue $1+i$ at $z=-1 / \zeta$ and residues totalling $(4 / m) S(\tau)$ at $z=\omega$ (the roots of equation (4)).

3. Some inequalities. We finally rearrange the terms of the sum $\sum \tau_{u}$ to display certain monotonicity properties. We ignore $m=8$ where the result follows from the fact that $\tau=2^{1 / 2}-1$ is a fundamental unit (or a slight variation of the main argument below). We introduce the new notations,

$$
\begin{aligned}
u & =(m / 4)-v-1, & \tau_{u} & =-\tan (4 v+3) \pi / m \\
u & =m / 8+w, & \tau_{u} & =-[\tan (4 w+1) \pi / m]^{-1}
\end{aligned}
$$

Then we write

$$
S(\tau, \lambda)=\sum_{u=0}^{m / 16-1} \phi
$$

where

$$
\phi=\gamma_{1}^{\lambda}-\gamma_{1}^{-\lambda}-\gamma_{2}^{\lambda}+\gamma_{2}^{-\lambda}
$$

with $\gamma_{1}=\tan (4 u+1) \pi / m$ and $\gamma_{2}=\tan (4 u+3) \pi / m$. Here, easily, $S(\tau, 1)=S(\tau)=-m / 4$ and if $\tau^{1 / q}=\rho$ were an integer in $R(\zeta+1 / \zeta)$, for an odd $q$, the trace of $\rho$ would be simply $S(\tau, 1 / q)$. Clearly a contra- 
diction would be achieved if we show that $S(\tau, \lambda)$ is always negative and increases monotonically from $-m / 4$ to 0 as $\lambda$ decreases from 1 to 0 .

We therefore let $\gamma_{1}$ and $\gamma_{2}$ be any two real constants satisfying

$$
0<\gamma_{1}<\gamma_{2}<1 \text {. }
$$

Then we finally establish the monotonicity of $\phi$ in $\lambda$ by writing

$$
\begin{aligned}
(d \phi / d \lambda) / \lambda & =\psi\left(G_{1}\right)-\psi\left(G_{2}\right), \\
\psi(G) & =(G+1 / G) \log G, \\
G_{i} & =\gamma_{i}^{\lambda}
\end{aligned}
$$$$
(i=1,2) \text {. }
$$

Thus we easily establish that $d \psi(G) / d G>0$ for $0<G<1$, whence $d \phi / d \lambda<0$ and $S(\tau, \lambda)$ has the required monotonicity.

4. Acknowledgments and alternate proof. The monotonicity property became apparent as the result of a tabulation of $S(\tau, 1 / q)$ made in August 1960 using the GEORGE computer at the Argonne National Laboratory, through the cooperation of Dr. William Miller, Applied Mathematics Division Director, and Miss Therese Czaja, programmer.

The referee has found the following purely algebraic proof which he has kindly consented to permit for inclusion:

Let $m=2^{k} \geqq 8, \zeta=\exp 2 \pi i / m$, and $\tau=\tan \pi / m=(\zeta-1) / i(\zeta+1)$. Suppose that $\tau=\alpha^{q}$ for odd $q>1$, and $\alpha$ in $R(\zeta+1 / \zeta)$. Since $i=( \pm i)^{q}$, then $\epsilon=i \tau=(i \beta)^{q}$ with $\beta= \pm \alpha$. Then $2 \zeta /(\zeta+1)=1+\epsilon=1+(i \beta)^{q}$ $=(1+i \beta)\left(1-i \beta+\cdots+(i \beta)^{q-1}\right)$. Since $2 \zeta /(\zeta+1)$ is a power of the prime ideal $\mathfrak{p}=(1-\zeta)$ in $R(\zeta)$ and since the last term is a product of two integers, $1+i \beta$ is also a power of $\mathfrak{p}$. Since $\mathfrak{p}$ is its own complex conjugate and $\beta$ is real, $1-i \beta / 1+i \beta$ is a unit of $R(\zeta)$ with absolute value 1 hence a root of unity in $R(\zeta)$. Let $1-i \beta / 1+i \beta=\zeta^{-a}$; then $i \beta=\zeta^{a}-1 / \zeta^{a}+1$. If $a$ were even, then $\tau=(i \beta)^{q} / i$ would be contained in $R\left(\zeta^{2}\right)$. Hence $a$ must be odd and there is an automorphism $\sigma$ of $R(\zeta)$ such that $\sigma(\zeta)=\zeta^{a}$. Then $i \beta=\sigma(\epsilon), \epsilon=\sigma(\epsilon)^{q}$ and $\sigma^{-1}(\epsilon)=\epsilon^{q}$. Since $\sigma^{m / 2}=1, \epsilon=\sigma^{-m}(\epsilon)=\epsilon^{q^{m}}$. Hence $\epsilon^{q^{m}-1}=1$, and $\tau=\epsilon / i$ must be a root of unity, which is clearly not true. Therefore $\tau \neq \alpha^{q}$.

\section{BIBLIOGRAPHY}

1. H. Cohn, A numerical study of Weber's real class number calculations. Part I, Numer. Math. vol. 2 (1960) pp. 347-362.

2. H. Weber, Lehrbuch der Algebra. II, Braunschweig, Friedrich Vieweg und Sohn Verlag, 1899, pp. 762-783.

University of Arizona 